\title{
Case Report: Management of Dengue Fever in the U.S.
}

Esther Bartlett, BS ${ }^{1}$; Albert Kim, BS ${ }^{1}$; Sudershan Singh, MD $^{2}$; Leonard Ranasinghe, MD, PhD ${ }^{\text {* }}$

${ }^{1}$ California North state University School of Medicine, 9700 W Taron Dr, Elk Grove, CA 95757, USA.

${ }^{2}$ Woodland Memorial Hospital, 1325 Cottonwood St, Woodland, CA 95695, USA.

*Corresponding Author: Leonard Ranasinghe, Professor of California Northstate University College of Medicine, 9700 W. Taron Dr. Elk Grove, CA 95757, USA.

Received Date: November 24, 2020; Accepted Date: December 28, 2020; Published Date: January 14, 2021. Citation: Bartlett E, Kim A, Singh S. and Ranasinghe L. (2021) Case Report: Management of Dengue Fever in the U.S., Clinical Medical Reviews and Reports, 3(1): DOI: 10.31579/2690-8794/053

Copyright: (C) 2021. Leonard Ranasinghe. This is an open-access article distributed under the terms of the Creative Commons Attribution License, which permits unrestricted use, distribution, and reproduction in any medium, provided the original author and source are credited.

\begin{abstract}
A patient diagnosed with dengue fever was recently treated at a hospital in California. This rare case raises the concern that dengue fever may become a larger threat in the future for the contiguous United States, which is not currently considered endemic for the disease. Over the past decade, there has been an increase in the number of cases; therefore, dengue fever must be given greater consideration in the differential diagnosis, especially with a patient travel history to endemic parts of the world. This case report examines the management of a patient presenting with dengue fever and demonstrates that continued vigilance, prompt testing, and patient education can improve patient care and decrease disease prevalence in the future.
\end{abstract}

Keywords: dengue fever; aedes aegypti; aedes albopictus; patient management; prevention

\section{Introduction}

Dengue fever is caused by an infection with one of the dengue flaviviruses: DENV-1, DENV-2, DENV-3, or DENV-4. All of them can cause dengue fever, and a patient can suffer from dengue fever repeatedly as they are subsequently infected with different DENV serotypes [1]. In fact, a patient's second illness is likely to be more severe than the first [2].

The viruses are carried by the Aedes aegypti and Aedes albopictus mosquitoes, which are also the vectors for Zika and Chikungunya. A female mosquito carrying the virus facilitates transmission as it takes its blood meal from a new host $[3,4]$. Local transmission is prevalent in tropical, subtropical, and urban environments where these mosquitoes exist, peaking during rainy seasons [5]. In recent years, the rising incidence of DENV infections has put approximately half of the world at risk [3]. In the US, studies show that A. aegypti has entered California over recent years, with 216 localized detections in 2019 [6], causing a return of dengue fever cases in California and other states [7, 8]. Current diagnostic methods include molecular tests, dengue virus antigen (NS1), serological tests, and fixed tissue tests [4], although there is a new Dengue vaccine approved by the FDA for use in endemic regions $[9,10]$.
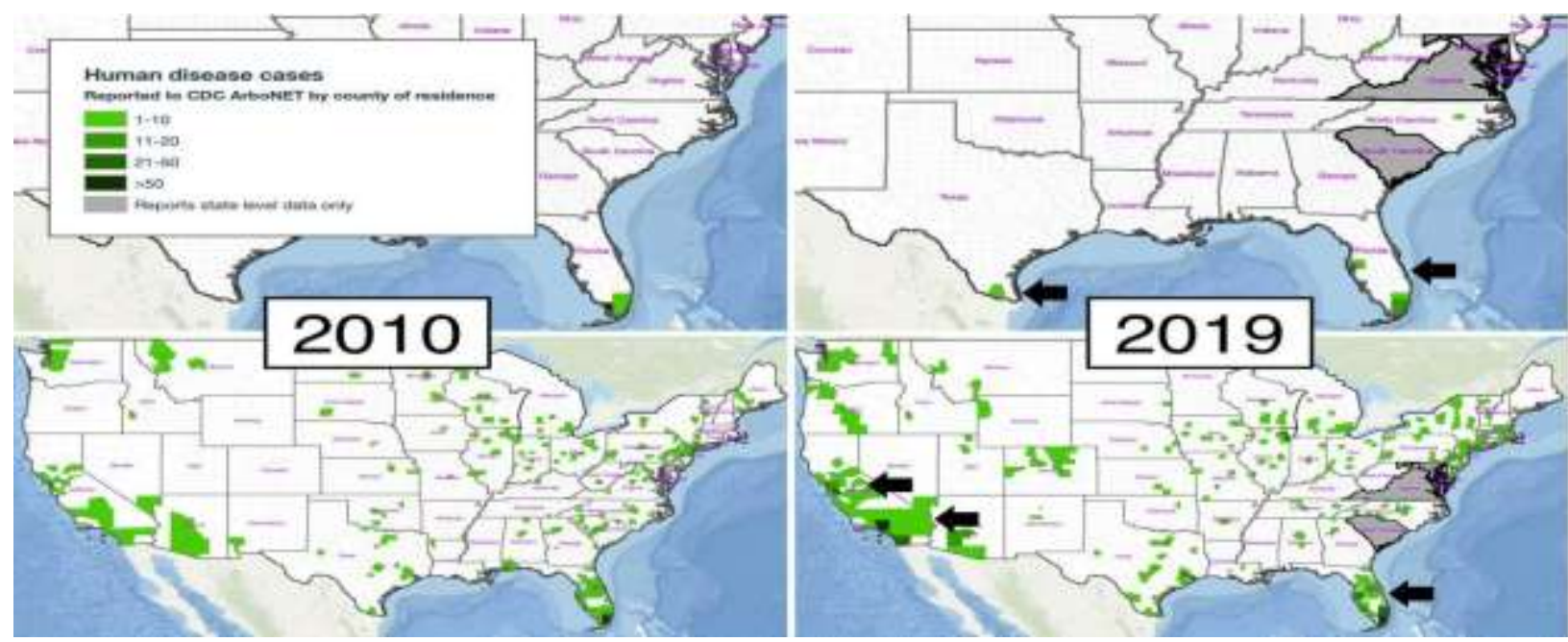
Figure 1. Increase in cases over the past decade. The two columns show data from the years 2010 and 2019 respectively. The top two images show local transmission and the bottom two images show imported cases. Black arrows show marked increase in reported cases in 2019 compared to 2010 [11].

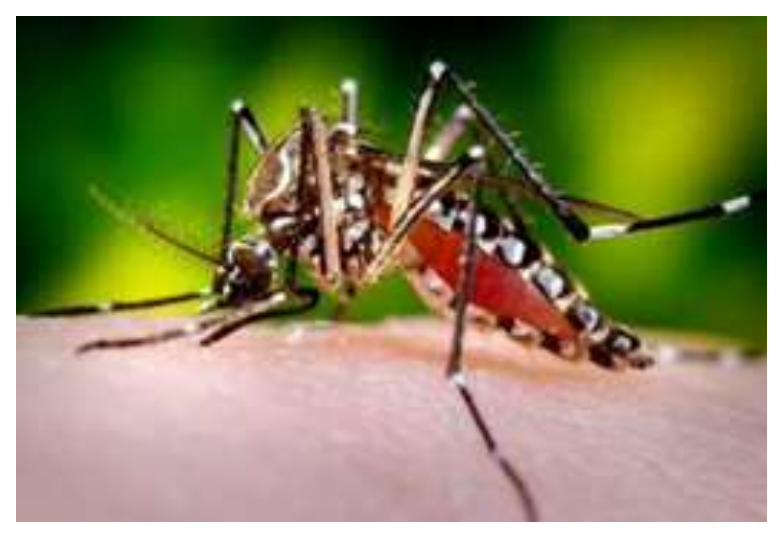

Figure 2a. The Aedes aegypti mosquito [4].
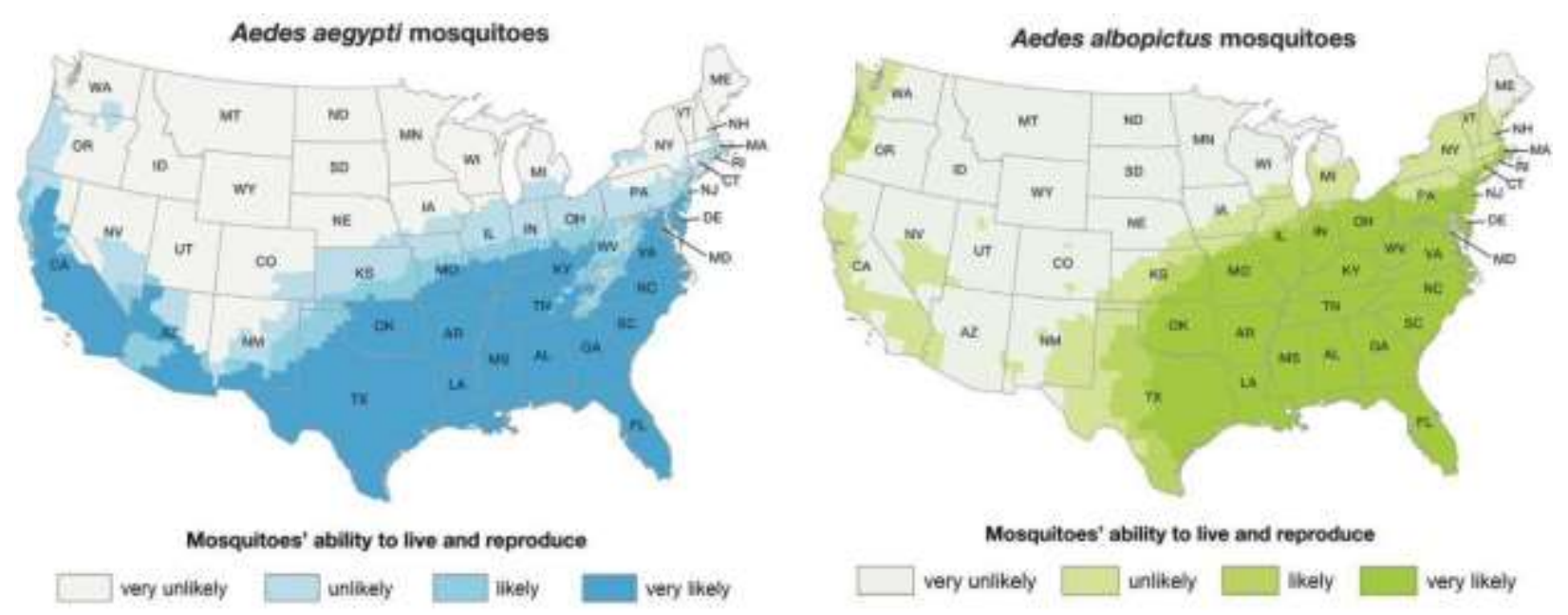

Figure 2b. Maps approximating the geographical presence of the Aedes mosquito. The presence of these mosquitoes makes local transmission possible [12].

\section{Case Report}

A 58-year-old female presented to the emergency department with a fiveday history of bilateral, "sharp" temporal headache $(8 / 10)$ radiating to the occiput. The patient also reported pruritic rashes on her upper and lower extremities and abdomen. She had nausea, vomiting, intermittent fever (100.4 ${ }^{\circ} \mathrm{F}$ at home), chills, body aches, light sensitivity, and phonophobia. The patient stated she returned from Mexico five days prior where she obtained numerous mosquito bites and was in close proximity with two family members who had active dengue fever. She was seen in urgent care two days ago for these symptoms, where she was prescribed Tamiflu, cetirizine, and triamcinolone topical; however, the patient did not take the medications. She was taking Advil and acetaminophen as needed. Past medical history is significant for prediabetes. The patient denied experiencing dysuria, hematuria, diarrhea, abdominal pain, shortness of breath, and chest pain. She also denied a history of liver or hematological problems.

On arrival, vital signs were: BP: $152 / 66 \mathrm{mmHg}$, pulse: $83 \mathrm{bpm}, \mathrm{RR}: 20$ breaths/min, Temperature: $36.6{ }^{\circ} \mathrm{C}$, BMI: $32.68 \mathrm{~kg} / \mathrm{m} 2$. Upon physical examination, the patient was alert but in pain. She appeared neurologically and hemodynamically stable. Although she kept her eyes closed, she did not have photophobia. The patient's neck had a full range of motion. She presented with petechiae on the posterior soft palate and both lower extremities; however, she no longer had the skin rash reported 2 days prior.

The patient was admitted to the hospital and the following lab tests were obtained: blood culture and coagulations, urine culture, complete blood count (CBC) with differentials, complete metabolic panel (CMP), liver function tests (LFT's), Serology for EBV and West Nile, Dengue Fever Abs IgG/IgM and head CT without contrast. Her white count and kidney function were normal. She had significant thrombocytopenia (platelets of 46), bandemia, leukocytosis, ketonuria, bilirubinemia, hematuria, and +2 bacteria. LFT's revealed elevated ALT (289), AST (573), and ALP (209). She had an elevated glucose level of $163 \mathrm{mg} / \mathrm{dL}$. The patient was found to have a urinary tract infection. She also had acute hepatitis, for which the physician ordered an additional acute hepatitis panel. The head CT was negative for bleeding, masses, or midline shift.

Following hospital admission, the patient was given Norco@ and Rocephin $\odot$ (Ceftriaxone) with procalcitonin. The next day, the infectious disease (ID) specialist diagnosed the patient with dengue fever, likely a second strain. Other differentials included Zika, Chikungunya, Malaria, West Nile virus, EBV, and HIV infections. The patient tested positive for hepatitis $\mathrm{C}$ with a negative ultrasound of the liver; the acute hepatitis 
appears to have been secondary to the dengue fever. For the dengue fever, she was treated with supportive IV fluids and closely monitored for hemorrhagic syndrome. She was given acetophaemin and told to avoid aspirin.

On the fourth day in the hospital, the patient was stable enough to be discharged for self- care at home. She was prescribed Oseltamivir (Tamiflu) $75 \mathrm{mg}$ oral capsule to take twice daily for 5 days, Cetirizine $10 \mathrm{mg}$ oral once daily, acetaminophen $500 \mathrm{mg}$ tablet every 4 hours as needed for fever, as well as a topical triamcinolone $0.1 \%$ cream to apply to the affected skin area 4 times daily. She was advised to maintain a normal diet and engage in physical activity as tolerable.

\section{Presentation/Signs and Symptoms}

Clinical presentations of the illness occur four to seven days after an infected bite and can range from asymptomatic to dengue hemorrhagic fever (DHF) and dengue shock syndrome (DSS), which can lead to death $[4,5]$. Most cases of DF are self-limited and present with sudden onset of fever, headache, retro-orbital pain, myalgia, arthralgia, and blanching, maculopapular rashes $[3,4]$. DHF classification requires hemorrhagic manifestations, thrombocytopenia, and plasma leakage, which often occur four to seven days after the initial onset of fever. DSS is marked by a weak pulse and narrow pulse pressure $(<20 \mathrm{~mm} \mathrm{Hg})$ in addition to the DHF criteria [13]. Rare manifestations include myocarditis, hepatitis [14], encephalopathy, and neuropathies [15].

\section{Differential Diagnoses}

Common differentials based on the history and febrile presentation of the illness include other flaviviruses, such as West Nile virus and Yellow Fever virus, malaria, chikungunya, typhoid fever, leptospirosis, measles, rubella, Epstein-Barr virus, rickettsial diseases, and early severe acute respiratory syndrome (SARS) [5]. Cross-reactions with other flaviviruses occur when performing an IgG ELISA, and rheumatoid factor may result in a false positive for dengue when performing an IgM ELISA [5].

\section{Management}

As the illness is self-limited and there is no specific therapy proven to reduce mortality rates, most cases are treated in the outpatient clinic with immediate fluid replacement, acetaminophen, and bed rest [16]. Aspirin and nonsteroidal anti-inflammatory drugs are contraindicated due to increased risk of bleeding. Conservative use of fresh-frozen plasma or fresh blood is indicated for hemorrhaging or disseminated intravascular coagulation. To monitor for DHF and DSS, platelet counts should be checked every 24 hours; patients should be admitted for intensive care if counts reach less than 100,000 per cubic millimeter [17].

\section{Discussion}

This case exemplifies the difficulty in diagnosing and promptly treating dengue fever in regions where it is less common. The patient was not given confirmation of her diagnosis even at her follow-up appointment a week after her discharge from the hospital. It is very important that the patient is aware of her infection so that she could properly protect against further infection with another dengue virus serotype, which could lead to more severe disease.

Because there is no specific treatment for dengue, the best way to combat it is through prevention of mosquito bites. Patients should be educated on the importance of using mosquito repellants like DEET and avoiding areas with large populations of mosquitoes. The majority of cases within the continental United States originated internationally; however, dengue fever is endemic in the US territories of Guam, Puerto Rico, U.S. Virgin Islands, and American Samoa. Given the progressive geographical spread of the Aedes aegypti and Aedes albopictus mosquito vectors, this case brings to question the potential risk of the dengue virus (DENV) becoming endemic in California and other regions in the United States. For future encounters with this virus, physicians should always inquire about travel history and consider dengue virus as a differential when it is indicated.

\section{Acknowledgement}

Special thanks to Ms. Brittany Alwis for editing this manuscript.

\section{References}

1. Halstead S. (2019) Recent advances in understanding dengue. F1000Research. 8.

2. Mustafa MS, Rasotgi V, Jain S, Gupta V. (2015) Discovery of fifth serotype of dengue virus (DENV-5): A new public health dilemma in dengue control. Medical journal armed forces India. 71(1):6770 .

3. World Health Organization. (2014) Dengue and severe dengue. World Health Organization. Regional Office for the Eastern Mediterranean.

4. Dengue. (2020) Centers for Disease Control and Prevention.

5. Wilder-Smith A, Schwartz E. (2005) Dengue in travelers. New England journal of medicine. 353(9):924-32.

6. Aedes aegypti and Aedes albopictus mosquitoes. California Department of Public Health. 2020 Nov 6.

7. Gloria-Soria A, Brown JE, Kramer V, Yoshimizu MH, Powell JR. (2014) Origin of the dengue fever mosquito, Aedes aegypti, in California. PLoS Negl Trop Dis. 8(7):e3029.

8. Pless E, Gloria-Soria A, Evans BR, Kramer V, Bolling BG, Tabachnick WJ, Powell JR. (2017) Multiple introductions of the dengue vector, Aedes aegypti, into California. PLoS neglected tropical diseases. 11(8):e005718.

9. First FDA-approved vaccine for the prevention of dengue disease in endemic regions. U.S. Food andDrug Administration. 2020 Nov 6.

10. Reynales H, Carrasquilla G, Zambrano B, Cortés M, Machabert T, et al. (2020) Secondary Analysis of the Efficacy and Safety Trial Data of the Tetravalent Dengue Vaccine in Children and Adolescents in Colombia. The Pediatric Infectious Disease Journal. 39(4):e30.

11. Dengue. ArboNET CDC. 2020 Nov 6.

12. Potential Range in US. Center for Disease Control and Prevention. 2020 Nov 6.

13. Jayaratne SD, Atukorale V, Gomes L, Chang T, Wijesinghe T, et al. (2012) Evaluation of the WHO revised criteria for classification of clinical disease severity in acute adult dengue infection. $B M C$ research notes. 5(1):645.

14. Lum LC, Lam SK, George R, Devi S. (1993) Fulminant hepatitis in dengue infection. The Southeast Asian journal of tropical medicine and public health. 24(3):467-71.

15. Sumarmo Wulur H, Jahja E, Gubler DJ, Sutomenggolo TS, Sulianti Saroso J. (1978) Encephalopathy associated with dengue infection. Encephalopathy associated with dengue infection. 44950.

16. Dung NM, Day NP, Tam DT, Loan HT, Chau HT, et al. (1999) Fluid replacement in dengue shock syndrome: a randomized, double-blind comparison of four intravenous-fluid regimens. Clinical infectious diseases. 29(4):787-94.

17. Gubler DJ. (1998) Dengue and dengue hemorrhagic fever. Clinical microbiology reviews. 11(3):480- 96. 